\title{
EDITORIAL
}

\section{Wilderness First Aid: Is it Time to Revisit Course Content?}

In this issue of Wilderness \& Environmental Medicine, Schuman et $\mathrm{al}^{1}$ present some information on knowledge gained, self-efficacy (self-rating of confidence about one's knowledge and skill level), and skills retention after a 16-hour Wilderness First Aid (WFA) course. They examined these 3 study criteria in a cross-section of 72 course participants at 4,8 , or 12 months after course completion.

This study has inherent experimental design limitations (participants came to only the first, second, or third examination periods) primarily due to the inability to logistically have participants return for all 3 examination periods after taking the course. Consequently, this is not a longitudinal assessment of retention based on a withinsubject experimental design, but rather, a cross-sectional group comparison. Even with this limitation, the reader of Wilderness \& Environmental Medicine has a great opportunity to gain insights on the key learning points about what knowledge and skills were retained across these 3 post-course examination periods.

These researchers reported that without regular use or refresher training, the participant's WFA knowledge and skills deteriorated as months increased from the initial course. These findings are not surprising and are predictable based on decades of research reporting the amount of skills retention after training, for courses such as Basic Life Support, Advanced Cardiac Life Support, and Advanced Trauma Life Support. Layperson cardiopulmonary resuscitation and automated external defibrillators skills rapidly deteriorate after the course. Consequently, it is reported that if people do act, they provide inferior quality cardiopulmonary resuscitation, such as chest compression depth and frequency, to patients in need. That, in turn, has been the rationale for the discussion of what are the best training methods and refresher (recertification) frequency, 1 or 2 years. These concepts certainly hold true for applying WFA skills to patients in the backcountry.

Wilderness First Aid is considered the "bread and butter" of all wilderness medicine courses taught in the United States, and was developed nearly 40 years ago by the organization that named this first-generation course the Mountain Rescue Seminar. Then, the course name was changed to Mountain Medicine, and eventually it became Wilderness First Aid, as it is taught by many organizations within the United States today. Originally, the course was designed for all outdoor enthusiasts so they could gain awareness and knowledge of how to manage common medical injuries, either for persons traveling alone or with family or friends. Today, the WFA content is used primarily by laypersons during short day trips or camps, weekend family activities, or any outdoor recreation. Currently, this course is taught to all ages, teens through seniors, nationally and internationally. At many organizations, this course is a requirement for seasonal and full-time camping staff, outdoor leaders and instructors, search and rescue personnel, and for members of the Boy Scouts for short outings and prolonged field trips. Some of the key WFA course content consists of performing a basic physical examination to identify obvious injuries or abnormalities, assessing signs and symptoms along with obtaining a simple relevant medical history, preventing medical problems anticipated by the activity and environment, initiating specific and appropriate medical treatments (basic splints, wound care, spine immobilization, managing heat and cold), and evaluating the need for appropriate type of evacuation.

Recent efforts among numerous educators, administrators, and organizations that have vested interests in wilderness medicine instruction have agreed to a WFA course framework with approximately 16 key core topics and as many as 5 elective topics. This course creates a robust amount of knowledge to assimilate along with subsequent skill application each day. That framework certainly poses challenges for both the instructor and student to meet course standards, and begs the question about the WFA course objectives. With so much content to cover over 2 days, can WFA graduates retain content and demonstrate correct skill application so that they will act confidently in a timely manner, not only during assessment in a research study but also in a demanding real-world application? The results from this study suggest that is not the case, particularly as time increases from initial training. Therefore, future studies are needed with a more robust within-subject research experimental design to confirm these findings. 
I am in agreement with the authors' conclusion that there should be a review of the high volume of WFA course topics. Currently, WFA course content does not allow a lot of quality time beyond basic skill demonstration and practice for the most common wilderness morbidities, such as soft tissue injuries, sprains, strains, fractures, and environmental exposure. Based on the authors' results and along with wilderness epidemiology literature, both resources provide insight for all who are vested in wilderness medicine training about what is the right amount and depth of WFA core content.

I applaud the efforts by Schumann et al, ${ }^{1}$ and I strongly encourage these authors and others, such as medical students and staff within U.S. and international wilderness medicine student interest groups, physicians in wilderness medicine fellowship programs, students and faculty in college recreation departments, and WFA educational organizations, to continue to team up to conduct and present similar research on WFA course topics, such as analyzing the difference in knowledge and skills retention between a Wilderness First Aid course and an Advanced Wilderness First Aid (4-day) course.

Brad L. Bennett, PhD, NREMT-P Wilderness Medical Society Secretary and Director, Academy of Wilderness Medicine Section Editor, Tactical and Operational Medicine, Wilderness \& Environmental Medicine Chair, Wilderness First Aid Practice Guidelines Working Group Bena, VA

\section{Reference}

1. Schumann SA, Schimelpfenig T, Sibthorp J, Collins RH. An examination of wilderness first aid knowledge, self-efficacy, and skill retention. Wilderness Environ Med. 2012;23: 281-287. 